\title{
IgAI dominant subclass of latent IgA mesangial deposition in donated kidney
}

Kazumasa Oka'

Kenji Nishimura ${ }^{2}$

Hidefumi Kishikawa ${ }^{2}$

Yasuji Ichikawa ${ }^{3}$

'Department of Pathology, Hyogo Prefectural Nishinomiya Hospital, Hyogo, Japan; ${ }^{2}$ Department of Urology, Hyogo Prefectural Nishinomiya Hospital, Hyogo, Japan; ${ }^{3}$ Department of Urology, Joyo Ejiri Hospital, Hyogo, Japan
Correspondence: Kazumasa Oka

Department of Pathology, Hyogo Prefectural Nishinomiya Hospital, Rokutanji-cho 13-9, Nishinomiya, Hyogo 662-0918, Japan

$\mathrm{Tel}+8 \mid 798345$ I5I

Fax +81798234594

Email oka1004@hp.pref.hyogo.jp
This article was published in the following Dove Press journal: International Journal of Nephrology and Renovascular Disease 29 November 2016

Number of times this article has been viewed

Background: In the pathogenesis of immunoglobulin A nephropathy (IgAN), the IgA1 subclass is more important than the IgA2 subclass. In healthy men, the prevalence of mesangial $\operatorname{IgA}$ deposition has been previously investigated. However, it remains unknown whether the presence of urinary abnormalities depends on the subclass of IgA deposition.

Materials and methods: We researched the subclasses of $\operatorname{IgA}(\operatorname{IgA} 1$ and $\operatorname{IgA} 2)$ by the direct immunofluorescence (IF) staining method using specimens in which we identified the deposition of IgA through zero-hour renal transplant biopsies from donors without urinary abnormalities. The samples of the zero-hour biopsies were collected from 46 cases of living renal transplant patients at Nishinomiya Hospital, Hyogo Prefecture, from January 2011 to December 2013.

Results: In seven of the 46 cases (15\%), IgA deposition and C3 in mesangium were confirmed. All seven cases showed IgA1 predominant mesangial deposition on IF. The results of the histological evaluations for all seven cases were Oxford Classification M0.S0.E0.T0.

Conclusion: This study showed similar patterns of latent mesangial IgA deposition according to IgA subclass and frequency of C3 deposition as IgAN. Latent mesangial IgA deposition may require some, as yet undefined factors, to become clinically apparent as IgAN.

Keywords: IgA nephropathy, IgA1, IgA subclass, latent, mesangium

\section{Introduction}

Immunoglobulin A nephropathy (IgAN) was first described by Berger and Hinglais in 1968 and is regarded as one of the most common forms of glomerulonephritis (GN) worldwide. End-stage renal disease is observed in $30 \%-40 \%$ of IgAN patients within 20 years of IgAN onset. ${ }^{1,2}$ It is characterized by predominant IgA deposits in the glomerular mesangium with $\mathrm{C} 3$ and/or IgG. Using light microscopy (LM), IgAN appears as mesangial proliferative $\mathrm{GN}$ and, on a case-by-case basis, may appear with endocapillary or extracapillary proliferation.

Human IgA can be classified into two subclasses $\operatorname{IgA} 1$ and $\operatorname{Ig} A 2$. Most investigators now agree that the IgA1 subclass is more critical than the IgA2 subclass in the pathogenesis of IgAN because most of the IgA deposited in the mesangium comprises IgA1 ${ }^{3,4}$ Recent studies strongly suggest that aberrant glycosylation of the $O$-linked glycans in the IgA1 hinge region is an important common pathogenic factor contributing to the development of IgAN. ${ }^{5-7}$

The major clinical presentation of $\operatorname{IgAN}$ is macroscopic and/or microscopic hematuria with proteinuria. However, in healthy men, the prevalence of mesangial $\operatorname{IgA}$ deposition has been previously investigated. Mesangial IgA deposition has been observed in $10 \%-30 \%$ of renal specimens through necropsy of patients without any 
manifestation of renal disease or in some donor kidneys during transplantation. ${ }^{8,9}$ This latent IgA deposition has not always been related to changes in glomerular inflammation.

It remains unknown whether the subclass of IgA deposition depends on the presence of urinary abnormalities and whether the deposition of the complement at IgAN can occur without urinary abnormalities. In addition, there is no report of the examination of a subclass of $\operatorname{IgA}$ in latent mesangial IgA deposition.

We researched the deposition of the subclasses of $\operatorname{IgA}$ using specimens in which we identified deposition of IgA in zero-hour renal transplant biopsies from donors with no urinary abnormalities.

\section{Materials and methods Materials}

The subjects of the present study were samples of zero-hour biopsies among the 46 living renal transplant patients at Nishinomiya Hospital, Hyogo Prefecture, from January 2011 to December 2013. Zero-hour allograft needle biopsies were immediately performed after reperfusion of kidneys removed from the donors.

\section{Methods}

Zero-hour renal biopsy specimens were divided for LM, immunofluorescence (IF) microscopy, and electron microscopy (EM). LM specimens were prepared in hematoxylin and eosin, Masson's trichrome, periodic acid-Schiff, and periodic acid methylamine silver. IF staining was performed as follows: zero-hour renal biopsy specimens were embedded in optimal cutting temperature compound, rapidly frozen in liquid nitrogen, and sectioned at $4 \mu \mathrm{m}$ with a cryostat. Direct IF staining was used to detect fluorescein isothiocyanateconjugated anti-human primary antibody (IgA, IgG, IgM, C3, C1q, Fibrinogen [Dako A/S, Copenhagen, Denmark; $\times 40$ ); IgA1 [Biorbyt, Cambridge, UK; orb22219, ×20]; and IgA2 [Biorbyt; orb22234, ×20]). The antibody was layered over the tissue and incubated for 30 minutes at room temperature, following which the slides were washed twice in phosphatebuffered saline. The slides were examined in a routine manner using an IF microscope. The intensity of fluorescence was graded as none $(-)$, trace $( \pm), 1(+), 2(+)$, and $3(+)$. The diagnosis of IgAN revealed the granular deposition of $>2(+)$ of IgA in the glomerular mesangium on IF, and the deposition of electron-dense material in the mesangium was confirmed through EM. Histological evaluation of LM was evaluated according to the Oxford Classification. ${ }^{10}$ The study protocol was approved by the ethics committee of Hyogo Prefectural Nishinomiya Hospital. The procedure for informed consent was that our study protocol was open to the public via our homepage (http://www.nishihosp.nishinomiya.hyogo.jp/ aboutus/rinshou kenkyu.html); if a participant did not want to be included in the study, they could make contact with us. There was no patient who did not want to be included in this study.

\section{Results}

$\operatorname{Ig}$ A deposition in mesangium was confirmed in seven out of 46 cases (15\%). All seven cases had no experience of urinalysis abnormalities or a history of secondary IgAN, such as alcoholic hepatic disease. In all seven cases, the dipstick method for testing urinary blood and proteinuria during the preoperative examination yielded negative results. Three out of the seven cases (43\%) had human leukocyte antigen (HLA)-DR4. Table 1 shows the clinical findings of the seven cases. Familial IgAN or Henoch-Schönlein purpura GN (HSPGN) was confirmed in five out of the seven cases. Table 2 shows the result of IF. All seven cases showed mesangial deposition of IgA and C3 on IF (Figure 1). The result of LM for all seven cases was Oxford Classification M0.S0.E0.T0 (Figure 2A). Mesangial proliferation was not observed in any of the cases. Electron-dense deposits in the mesangial area were confirmed in all seven cases using EM (Figure 2B). All seven cases showed IgA1 predominant mesangial deposition on IF (Figure 3 ).

Table I Clinical data of seven patients

\begin{tabular}{lllllll}
\hline Pt & Age (years) & $\begin{array}{l}\text { Relationship with } \\
\text { recipient }\end{array}$ & Primary disease of recipient & HLA-DR4 & eGFR (mL/min/l.73 $\left.\mathbf{~ m}^{2}\right)$ & History of hypertension \\
\hline I & 63 & Mother & IgAN & + & 67.7 & + \\
2 & 33 & Brother & IgAN & - & 96 & - \\
3 & 38 & Sister & HSPGN & - & 66.2 & - \\
4 & 68 & Mother & HSPGN & + & 47.8 & + \\
5 & 53 & Mother & Unknown & + & 82.2 & - \\
6 & 71 & Mother & IgAN & - & 92.1 & - \\
7 & 65 & Mother & Unknown & - & 51.7 & - \\
\hline
\end{tabular}

Abbreviations: Pt, patient; HSPGN, Henoch-Schönlein purpura glomerulonephritis; eGFR, estimated glomerular filtration rate; IgAN, immunoglobulin A nephropathy; HLA-DR4, human leukocyte antigen-DR4. 
Table 2 IF staining findings of seven patients

\begin{tabular}{|c|c|c|c|c|c|c|c|c|}
\hline $\mathbf{P t}$ & |gA| & $\operatorname{IgA2}$ & $\operatorname{IgA}$ & IgG & IgM & C3 & $\mathrm{CIq}$ & Fibrinogen \\
\hline I & ++ , mes & - & +++ , mes & - &,+ mes & + , mes & - & - \\
\hline 2 & ++ , mes & - & +++ , mes & - & - & + , mes & - & - \\
\hline 3 & ++ , mes & - & +++ , mes & - &,+ mes & \pm , mes & - & - \\
\hline 4 & ++ , mes & - & +++ , mes & - & - & + , mes & - & - \\
\hline 5 & ++ , mes & - & +++ , mes & - & - & + , mes & - & - \\
\hline 6 & ++ , mes & - & ++ , mes & - & - & + , mes & - & - \\
\hline 7 & ++ , mes & - & ++ , mes & - & - & + , mes & - & - \\
\hline
\end{tabular}

Abbreviations: Pt, patient; IF, immunofluorescence; mes, mesangium; Ig, immunoglobulin.
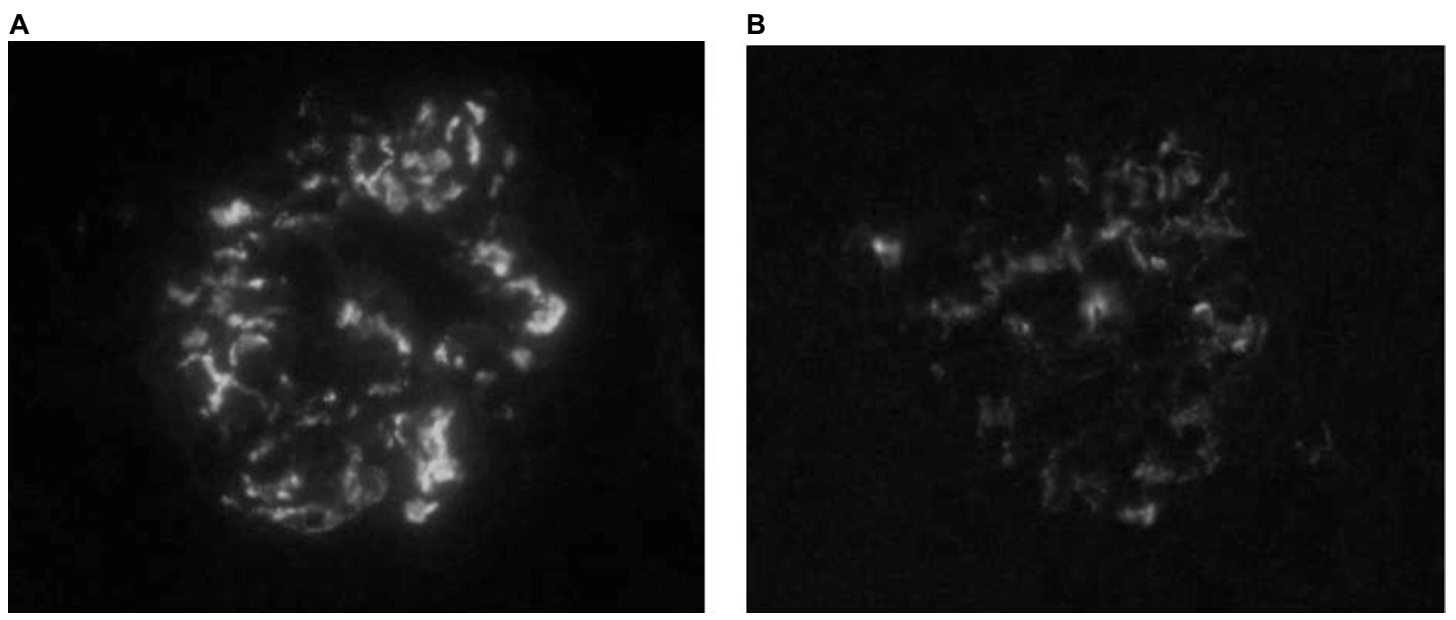

Figure I Staining by IF using frozen sections of zero-hour renal allograft biopsy.

Note: IgA (A) and C3 (B) reveal mesangial deposition, respectively, $(+++),(++)$. Magnification $\times 400$.

Abbreviations: IF, immunofluorescence; IgA, immunoglobulin A.

A

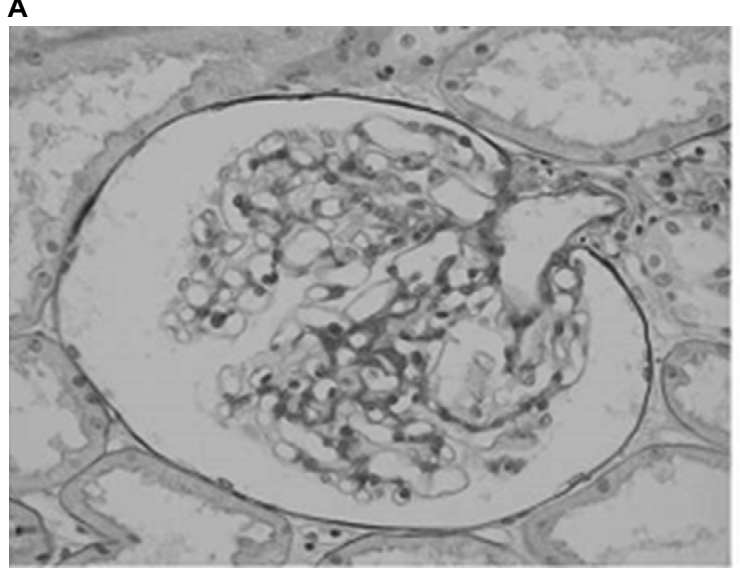

B

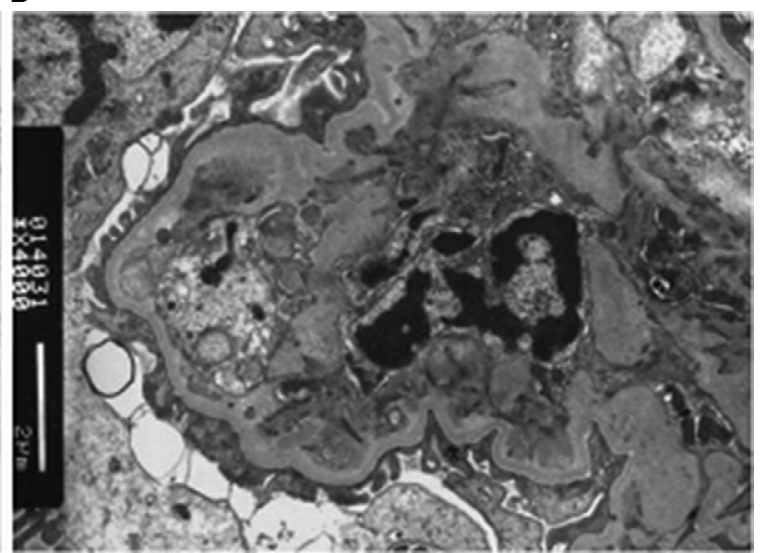

Figure 2 Histological findings of zero-hour renal allograft biopsy.

Notes: Biopsy specimen shows minor glomerular abnormalities (periodic acid-Schiff) (A). By electron microscopy, mesangial lgA deposition is confirmed (B). Magnification $\times 400$.

Abbreviation: $\lg A$, immunoglobulin $A$.

\section{Discussion}

In the present study, we made two important clinical observations. First, we found latent $\operatorname{IgA}$ mesangial deposition of the IgA1 subclass to be more dominant than that of the IgA2 subclass. Second, we confirmed familial IgA nephropathy or HPSGN in five of seven cases with latent IgA mesangial deposition.
We found latent IgA mesangial deposition of the IgA1 subclass to be more dominant than that of the IgA2 subclass. In majority of the studies, IgA deposited in the glomeruli of IgAN was shown to be predominantly of the IgA1 subclass. ${ }^{3,4}$ Our results of latent IgA mesangial deposition were consistent with the results of several previous studies of IgAN. Recent data suggest that aberrant glycosylation of the 
A

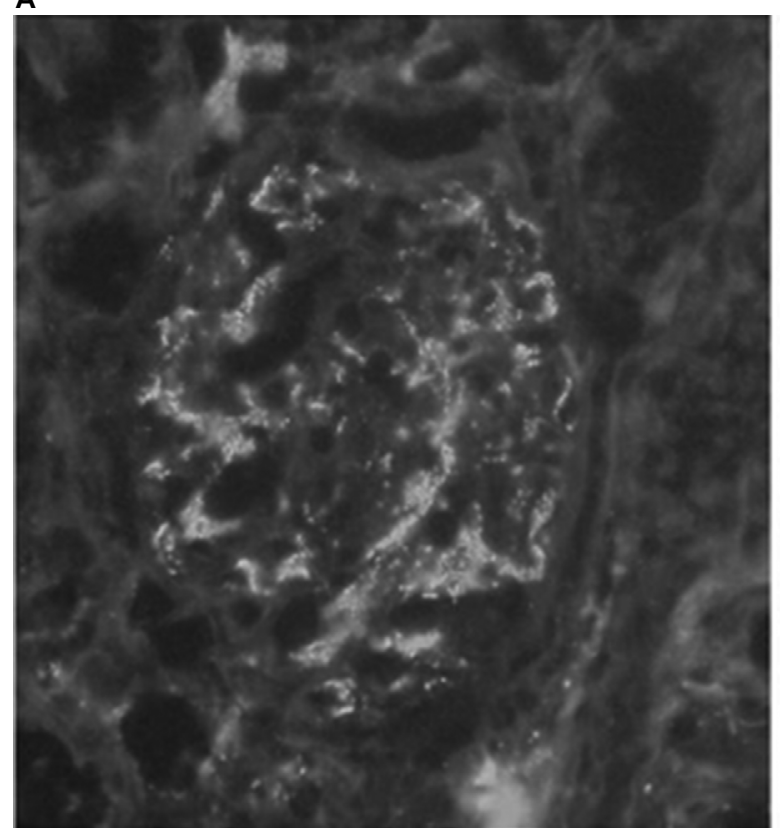

B

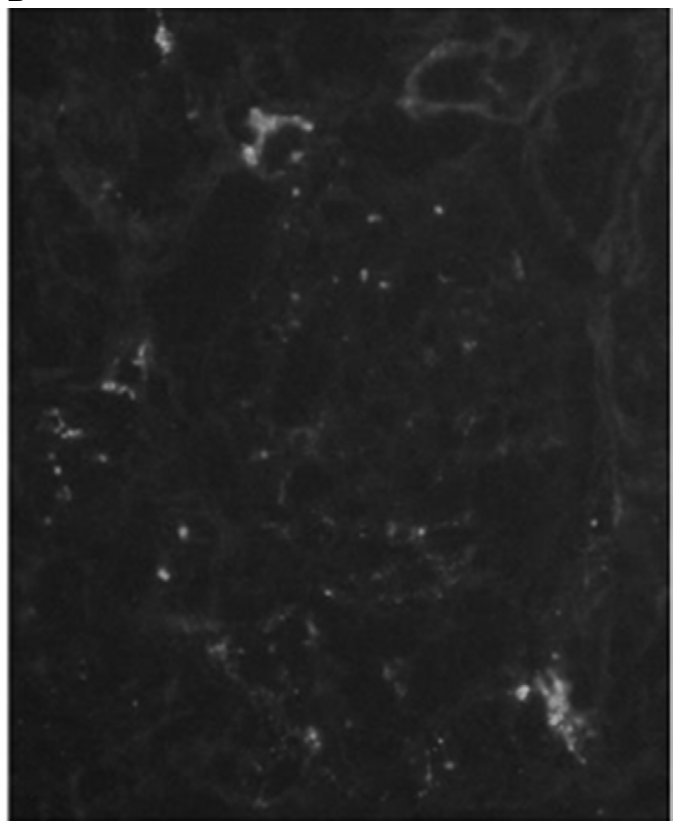

Figure 3 Staining by IF using frozen sections of zero-hour renal allograft biopsy.

Notes: IgAI reveals mesangial deposition $(++)($ A). $\lg A 2$ is no staining $(-)$ (B). Magnification $\times 400$

Abbreviations: IF, immunofluorescence; IgA, immunoglobulin $A$.

$O$-linked glycans in the IgA1 hinge region is a key factor in the development of IgAN. ${ }^{5-7}$ In future, we plan to determine whether latent IgA mesangial deposition is associated with aberrant glycosylation of the $O$-linked glycans in the $\operatorname{IgA} 1$ hinge region.

Second, we confirmed familial IgA nephropathy or HPSGN in five out of seven cases with latent IgA mesangial deposition. The first documentation of familial cases of biopsy-proven IgAN was in two HLA-identical brothers in $1978 .{ }^{11}$ The younger brother developed end-stage renal disease and received a kidney transplant from the brother who had normal renal function. A preoperative biopsy of the donor kidney showed minimal histological abnormalities; however, mesangial deposits of IgA and C3 on IF and mesangial deposits on EM were apparent. Some reports regarding the prognosis of familial IgAN exist. Familial IgAN limited to a single generation (suggestive of autosomal recessive inheritance) has a significantly poor outcome compared with that involving two or more generations (suggestive of autosomal dominant inheritance). ${ }^{12}$ However, previous studies failed to confirm that familial IgA nephropathy has a prognosis worse than its sporadic form. ${ }^{13}$ Familial IgAN is thought to be genetically heterogeneous. The reason for a different prognosis may be seen in familial IgAN. A strong association between HLA-DR4 with benign IgAN was reported. ${ }^{14}$ In the present study, three out of seven cases (43\%) had HLA-DR4.
In the present study, latent IgA mesangial deposition was found to be associated with minor glomerular abnormalities through LM. Incidental mesangial IgA deposition has been reported in $10 \%-30 \%$ of renal specimens through necropsy of patients without any manifestation of renal disease or in some donor kidneys during transplantation. ${ }^{8,9}$ These incidental cases of IgA deposition have not always been related to changes in glomerular inflammation. These results suggest that IgA or IgA1 can be deposited in the mesangium without overt glomerular damage. The deposition of IgG in IgAN was associated with mesangial and endocapillary hypercellularity, showing a trend toward worse renal survival. ${ }^{15,16}$ All of our seven cases with latent IgA mesangial deposition showed no IgG mesangial deposition. IgG may be one of the linking factors between subclinical IgA deposition and IgAN. C3 is detected in glomeruli in $>90 \%$ of cases of IgAN. ${ }^{17}$ Varis et al ${ }^{18}$ stated that the complement appears to be one of the linking factors between the deposition of subclinical IgA and IgAN, because their study showed isolated $\operatorname{IgA}$ deposition without $\mathrm{C} 3$ in cases of suicide or violent death. We found mesangial deposition of $\mathrm{C} 3$ in cases with latent IgA mesangial deposition. There may be other factors linking subclinical IgA deposition and IgAN.

\section{Conclusion}

The present study showed consistent results of mesangial deposition according to IgA subclass and frequency of 
C3 deposition as IgAN. Asymptomatic IgAN may require some as yet undefined factors to become clinically apparent as IgAN.

\section{Disclosure}

The authors report no conflicts of interest in this work.

\section{References}

1. Berger JH. Intercapillary deposits of IgA-IgG. J Urol Nephrol. 1968;74(9):694-695.

2. D'Amico G. Influence of clinical and histological features on actuarial renal survival in adult patients with idiopathic IgA nephropathy, membranous nephropathy, and membranoproliferative glomerulonephritis: survey of the recent literature. Am J KidneyDis. 1992;20(4):315-323.

3. Conley ME, Cooper MD, Michael AF. Selective deposition of immunoglobulin A1 in immunoglobulin A nephropathy, anaphylactoid purpura nephritis, and systemic lupus erythematosus. $J$ Clin Invest. 1980;66(6):1432-1436.

4. Suzuki S, Kobayashi H, Sato H, Arakawa M. Immunohistochemical characterization of glomerular IgA deposits in IgA nephropathy. Clin Nephrol. 1990;33(2):66-71.

5. Hiki Y, Odani $\mathrm{H}$, Takahashi M, et al. Mass spectrometry proves under-Oglycosylation of glomerular IgA1 in IgA nephropathy. Kidney Int. 2001; 59(3):1077-1085.

6. Allen AC, Bailey EM, Brenchley PE, Buck KS, Barratt J, Feehally J. Mesangial IgA1 in IgA nephropathy exhibits aberrant O-glycosylation: observations in three patients. Kidney Int. 2001;60(3):969-973.

7. Lai KN. Pathogenesis of IgA nephropathy. Nat Rev Nephrol. 2012; 20(5):275-283.

8. Sinniah R. Occurrence of mesangial IgA and IgM deposits in a control necropsy population. J Clin Pathol. 1983;36(3):276-279.
9. Suzuki K, Honda K, Tanabe K, et al. Incidence of latent mesangial IgA deposition in renal allograft donors in Japan. Kidney Int. 2003;63(6): 2286-2294.

10. Working Group of the International IgA Nephropathy Network and the Renal Pathology Society, Roberts IS, Cook HT, et al. The Oxford classification of IgA nephropathy: pathology definitions, correlations, and reproducibility. Kidney Int. 2009;76(5):546-556.

11. Tolkoff-Rubin NE, Cosimi AB, Fuller T, Rubin RH, Colvin RB. IGA nephropathy in HLA-identical siblings. Transplantation. 1978;26(6): 430-433.

12. Schena FP, Cerullo G, Rossini M, Lanzilotta SG, D'Altri C, Manno C. Increased risk of end-stage renal disease in familial $\operatorname{IgA}$ nephropathy. J Am Soc Nephrol. 2002;13(2):453-460.

13. Tam KY, Leung JC, Chan LY, Lam MF, Tang SC, Lai KN. Macromolecular IgA1 taken from patients with familial IgA nephropathy or their asymptomatic relatives have higher reactivity to mesangial cells in vitro. Kidney Int. 2009;75(12):1330-1339.

14. Hiki Y, Kobayashi Y, Tateno S, Sada M, Kashiwagi N. Strong association of HLA-DR4 with benign IgA nephropathy. Nephron. 1982;32(3) 222-226.

15. Bellur SS, Troyanov S, Cook HT, Robert IS. Working Group of International IgA Nephropathy Network and Renal Pathology Society. Immunostaining findings in IgA nephropathy: correlation with histology and clinical outcome in the Oxford classification patient cohort. Nephrol Dial Transplant. 2011;26(8):2533-2536.

16. Wada Y, Ogata H, Takeshige Y, et al. Clinical significance of IgG deposition in the glomerular mesangial area in patients with IgA nephropathy. Clin Exp Nephrol. 2013;17(1):73-82.

17. Nakagawa H, Suzuki S, Haneda M, Gejyo F, Kikkawa R. Significance of glomerular deposition of $\mathrm{C} 3 \mathrm{c}$ and $\mathrm{C} 3 \mathrm{~d}$ in $\mathrm{IgA}$ nephropathy. Am J Nephrol. 2000;20(2):122-128.

18. Varis J, Rantala I, Pasternack A, et al. Immunoglobulin and complement deposition in glomeruli of 756 subjects who had committed suicide or met with a violent death. $J$ Clin Pathol. 1993;46(7):607-610.

\section{Publish your work in this journal}

The International Journal of Nephrology and Renovascular Disease is an international, peer-reviewed open access journal focusing on the pathophysiology of the kidney and vascular supply. Epidemiology, screening, diagnosis, and treatment interventions are covered as well as basic science, biochemical and immunological studies. The manuscript management system is completely online and includes a very quick and fair peer-review system, which is all easy to use. Visit http://www. dovepress.com/testimonials.php to read real quotes from published authors. 